Restrepo. D. y Cárdenas, R. (2020), Caracterización de la internacionalización del currículo contable, caso programas de Contaduría Pública IUE y Unaula. Contaduría Universidad de Antioquia, 76, 159-185.

Doi: https://doi.org/10.17533/udea.rc.n76a08

\title{
Caracterización de la internacionalización del currículo contable, caso programas de Contaduría Pública IUE y Unaula*
}

\author{
Diego Fernando Restrepo Duque \\ diego.restrepodu@unaula.edu.co \\ Universidad Autónoma Latinoamericana \\ orcid: 0000-0001-9769-5512
}

Rogelio Cárdenas Londoño rcardenas@correo.iue.edu.co Institución Universitaria de Envigado orcid: 0000-0002-5015-1427

Propuesta que emerge como resultado del proyecto de investigación ejecutado, titulado: Orientaciones para la internacionalización de los procesos curriculares en las Instituciones de Educación Superior, caso programas de contaduría pública Institución Universitaria de Envigado, IUE, y la Universidad Autónoma Latinoamericana, UNAULA. 
Caracterización de la internacionalización del currículo contable, caso programas de Contaduría Pública IUE y Unaula

Resumen: Este texto sobre la armonización de los currículos contables tiene como propósito presentar una caracterización de las diferentes formas de internacionalización de los currículos que se han encontrado en los diversos reportes que se hacen desde los programas de contaduría pública, y finalmente, proponer ideas que puedan ayudar a diseñar estudios desde un enfoque conceptual y metodológicamente apropiados. Este proceso investigativo se asume desde un estudio descriptivo, basado en recolección de información a través de fuentes primarias, analizadas por métodos de estadística descriptiva, matrices de proximidad y correlación, y contrastada a través de entrevistas a profundidad. Uno de los productos de este proyecto es la integración, síntesis e interpretación de estos efectos que trae consigo la internacionalización para la disciplina contable.

Palabras clave: Armonización curricular, Ciclo de calidad, Gestión de conocimiento, Internacionalización del currículo, Movilidad, Multiculturalidad.

Characterization of the internationalization of the accounting curriculum; the case of Public Accounting programs at IUE and Unaula

Abstract: This paper on the harmonization of the accounting curricula aims at presenting a characterization of the different ways of curricula internationalization found in the different reports made from the public accounting programs; to then propose ideas that can help design methodologically appropriate studies from a conceptual approach. This research process is undertaken from a descriptive study, based on information gathering through primary sources, analyzed with descriptive statistics methods, proximity and correlation matrices, and contrasted through in-depth interviews. One of the products of this project is the integration, synthesithis and interpretation of these effects brought about by internationalization for the accounting discipline.

Keywords: Curricular harmonization, Quality cycle, Knowledge management, Internationalization of the curriculum, Mobility, Multiculturalism.

Caraterização da internacionalização do currículo contábil, caso programas de Contabilidade Pública IUE e Unaula

Resumo: Este texto sobre a harmonização dos currículos contábeis tem como objetivo apresentar uma caracterização das diferentes formas de internacionalizar os currículos que tem se encontrado nos diversos reportes que se elaboram desde os programas de contabilidade pública; e finalmente, propor ideias que possam ajudar a desenhar estudos desde uma ótica conceitual e metodologicamente apropriados. Este processo de pesquisa se aborda desde um estudo descritivo, baseado na recoleção de informação através de fontes primárias, analisadas por métodos de estatística descritiva, matrizes de proximidade e correlação, e contrastada através de entrevistas em profundidade. Um dos produtos deste projeto é a integração, síntese e interpretação destes enfoques que traz consigo a internacionalização para a disciplina contábil.

Palavras-chave: Harmonização curricular, ciclo de qualidade, gestão do conhecimento, internacionalização do currículo, mobilidade, multiculturalidade.

Un caractérisation de l'internationalisation du programme comptable, le cas des programmes de comptabilité publique de I 'institution Universitaire d'Envigado -IUE et d'Unaula en Colombie

Résumé : ce texte sur l'armonisation des programmes comptables a pour but de présenter une caractérisation des différentes formes d'internationalisation des programmes trouvées dans plusieurs rapports faits par les programmes de comptabilité publique. Il a aussi pour but de proposer des idées qui puissent aider dans la conception d'études basées sur une approche conceptuelle et méthodologique apropriée. Ce processus de recherche est abordé à partir d'une étude descriptive, basée sur le rassemblement d'information à travers des sources primaires, analysées avec des méthodes de statistique descriptives, matrices de proximité et de corrélation, et comparé au moyen d'entretiens approfondies. Un des produits de ce projet est l'intégration, la synthèse et l'interprétation des effets que comporte l'internationalisation pour la discipline comptable.

Mots clés : armonisation des programmes, cycle de qualité, gestion des connaissances, internationalisation du programme, mobilité, muti-culturalité 


\title{
Caracterización de la internacionalización del currículo contable, caso programas de Contaduría Pública IUE y Unaula
}

\author{
Diego Fernando Restrepo Duque y Rogelio Cárdenas Londoño \\ https://doi.org/10.17533/udea.rc.n76a08
}

Primera versión recibida en diciembre de 2019 - Versión aceptada en mayo de 2020

\section{Introducción}

$\mathrm{H}$

oy existe una fuerte expansión del proceso de internacionalización de la educación superior que es apropiada por numerosas instituciones que tienen entre sus objetivos dinamizar factores que coadyuven a garantizar la calidad universitaria y en hacer de la universidad esa organización clave que fomenta y difunde el conocimiento universal, a partir de acoger actores sociales propios de las comunidades que se albergan a su interior; comunidades académicas y comunidades científicas, las que están en permanente sintonía con sus pares y amigos en otros centros generadores de conocimiento en las distintas ramas del saber científico.

Existen dos conceptos clave que estructuran teóricamente este proceso de la internacionalización: la movilidad y la cooperación; movilidad tanto de personas como de afectos, prácticas, valores, concepciones y modos de vivir en un ambiente como el que generan las IES en todos los rincones de las distintas geografías soportados en convenios, en alianzas y en acuerdos que permiten la viabilidad operativa de todo este andamiaje conceptual, teórico y metodológico.

Si se sopesa el concepto de movilidad y su trazabilidad histórica, se ve su inicio en contextos internos e intramuros con marcas significativas de rápidos y fuertes aumentos en las cifras o datos cuantitativos e igualmente en fenómenos cualitativos que la soportaron desde las instituciones de origen hasta las de destino, el caso de mayor impacto se refleja en el programa Erasmus Mundus que ha cambiado la realidad de la dimensión internacional en las universidades europeas y ha creado condiciones necesarias para fases sucesivas, partiendo de infraestructuras de apoyo a la movilidad, con mejores oficinas dedicadas a 
Restrepo. D. y Cárdenas, R. Caracterización de la internacionalización del currículo contable...

estos menesteres, con una categoría de profesionales de la movilidad, y a partir de esto se ha venido acumulando experiencias que permiten ser replicadas en otros contextos y que nos sirve de referencia para lo que se configura en este texto.

Fruto de estos esfuerzos sistematizados y viabilizados se encuentran programas tales como: Tempus con la Europa del Este y la ex Unión Soviética, y más tarde con la cuenca del Mediterráneo; programa Alfa con América Latina; programa Atlantis con los Estados Unidos, y más recientemente el programa Erasmus Mundus por la cooperación entre universidades europeas y de cualquier otro país del mundo en los marcos de los estudios posgraduales tal y como lo sostiene Guy Haug (2010).

Existen otros procesos como es el caso Bolonia (1999), fruto de la integración europea, y la estrategia de Lisboa (2002), y la Declaración de Salamanca (2001), entre otros, que han fomentado la internacionalización.

\section{Referente conceptual y teórico}

El concepto de currículo lo asociamos al término tan conocido por todos Curriculum vitae, en el cual se recogen las diferentes vivencias profesionales que ha ido adquiriendo la persona; en este caso la hoja de vida de profesores, profesionales, su formación y experiencia. Una segunda acepción es la del currículo en el campo de las disciplinas de la educación al lado de la filosofía, piscología, antropología, sociología, epistemología, pedagogía, didáctica, evaluación, investigación, las TIC, segundo idioma, en círculo virtuoso de formación, perfeccionamiento, desempeño investigativo y de calidad de un educador, en cualquiera de los niveles del sistema educativo, de preescolar a posdoctorados, tal y como lo afirma Galeano (2016, p. 37).

El término currículo es muy antiguo, procede del vocablo latino currere, que significa carrera. Se refiere al recorrido realizado por una persona, un profesional. Son válidas las dos acepciones: la hoja de vida y la apropiación en el campo de las disciplinas de la educación.

Los referentes teóricos que orientan esta búsqueda por armonizar los currículos de los programas académicos contables de la Universidad Autónoma Latinoamericana, Unaula y de la Institución Universitaria de Envigado, IUE, se basan en autores como Bobbit Franklin (2008), Tyler Raph (1949), Bloom Benjamin (1976), Meyer Robert (1961), Bruner Jerome (1961), Taba Hilda (1991) y Tyler Federich W. (1971), desde la escuela del Racionalismo Instrumental.

En consonancia con esta búsqueda se hace necesario asumir la teoría hermenéutica dialéctica, desde sus mejores representantes, Gauntier (1963), Jackson (1968), Schwab (1969), Habermas (1973, 1979), Gadamer (1975, 1979), Stenhouse (1975), Eliott (1980), Schon (1992), Pérez, G. (1983), Reid (1985), Carr y Kemmis (1986), Goodson (2000), entre otros. 
Hoy se hace mayor conciencia de las múltiples modificaciones que ha sufrido el currículo para conservar su esencia. Su metamorfosis ha sido de un currículo por contenidos a un currículo por objetivos, estándares, indicadores y últimamente por competencias, sin llegar aún al gran cambio, conservándose en el currículo impulsado por el racionalismo instrumental para una sociedad industrial.

En términos de la internacionalización curricular, una pretensión desde el marco conceptual y teórico, nos acerca a quienes sostienen que la internacionalización del currículo que se ha implementado en las Instituciones de Educación Superior, IES, es favorable a la movilidad (Ortiz, 2015); (De Zan, 2011); (Ramírez Lozano, 2013); (Guido y Guzmán, 2012); (Tangelson, 2014); (Gacel, 2008); de igual forma, otras voces autorizadas, precisan su concepción y la podemos obtener de (Hawawini, 2011) quien señala que la internacionalización en las instituciones de educación superior es un proceso de integración de actores básicos y sus principales grupos de interés (estudiantes, profesores, y personal administrativo).

En igual sentido (Gacel, 2008) sostiene que todo proceso de internacionalización se construye y desarrolla a partir de la situación propia de cada sistema de educación superior, de sus fuerzas y debilidades, la cuales tienen el potencial y la viabilidad de las estrategias pertinentes, de cara al fortalecimiento de la calidad de la educación.

Por su lado Aponte (2016), dice que el currículo internacional es la expresión de los elementos que interactúan en una institución en correspondencia con su naturaleza e identidad y cuando se han integrado las dimensiones intercultural, internacional y multidisciplinar, para formar personas capaces de interactuar social y profesionalmente en un contexto global.

Esto nos permite criterios de fundamentación con base en referentes internacionales, conceptualización del programa, perfil profesional, título que se otorga, y estructura del plan de estudios.

Cuando se analiza su implementación en la Universidad Autónoma Latinoamericana, Unaula, y en la Institución Universitaria de Envigado, IUE, se ve su transición que transita de un currículo por contenidos y su desarrollo curricular de clase magistral y evaluaciones memorísticas, hasta un currículo técnico profesionalizante por objetivos, con el Decreto 080 de 1980; posteriormente, con la Ley 30 de 1992, se asume dentro del modelo educativo de formación integral, libre desarrollo de la personalidad, libertad de cátedra y de investigación; por último, la Ley 1188 de 2008 y su Decreto 1295 de 2010 que obliga el paso del currículo tradicional por contenidos y técnico por objetivos, al currículo por competencias, dentro del enfoque del racionalismo instrumental y el positivismo que permea en todas sus vertientes. 
Restrepo. D. y Cárdenas, R. Caracterización de la internacionalización del currículo contable...

\section{Hipótesis y supuestos}

Son los tipos de estrategias, técnicas y métodos curriculares actuales los que se deben establecer para identificar las necesidades de perfeccionamiento y acompañamiento académico y técnico de las diferentes áreas de la organización curricular, con sus respectivos componentes y asignaturas, las que estructuran el plan de estudios de los programas académicos de la Universidad Autónoma Latinoamericana, Unaula, y de la Institución Universitaria de Envigado, IUE.

\section{Metodología}

El interés centrado en este proceso investigativo se ubica en la armonización del currículo; su área de influencia abarca a las Instituciones de Educación Superior que sirven de referencia y que integran el caso bajo la concepción de Yin (2009); a saber: Universidad Autónoma Latinoamericana, Unaula, y la Institución Universitaria de Envigado, IUE; dos instituciones de educación superior - privada la primera y pública municipal, la segunda- que hacen parte de RedSicon, Red de investigadores y Semilleros del campo contable, cuya práctica orienta un interés común por valorar la formación de los planes de estudio de los programas académicos de Contaduría Pública que se encuentran en el valle de Aburrá, lo que permitió formular el siguiente interrogante, bajo la concepción de las comunidades de indagación planteadas por Cárdenas (2016): ¿Qué tipo de prácticas, estrategias, técnicas y métodos nos ayudan a caracterizar las diferentes formas de internacionalización de los currículos que deben operar en los programas de formación académica en las Instituciones de Educación Superior y que sirven de referente para la acreditación institucional a emprender; y con marcos de referencia conceptual y metodológica correctos?

Detrás de esta pregunta ronda el deseo por querer presentar una caracterización de las diferentes formas de internacionalización de los currículos que se han encontrado en los variados reportes que se hacen desde los programas de Contaduría Pública cuando se asumen los procesos de acreditación que orienta el CNA (2013), Consejo Nacional de Acreditación, a la vez que se busca poder desprender pautas y orientaciones didácticas para guiar la utilización de los procesos de internacionalización del currículo y su concreción en procesos de armonización.

Siguiendo indicaciones consensuadas entre los participantes en el proceso investigativo, se realizó la selección de la muestra basada en criterios, de acuerdo con la exigencia de determinar por adelantado un conjunto de atributos que debía poseer la unidad de estudio. Los criterios para la selección de la muestra de las instituciones de educación superior con las que se adelantaría la investigación fueron los siguientes: 
- Que estuvieran vinculadas al Sistema Nacional de información de educación superior, SNIES.

- Que la experiencia se realizara bajo un enfoque de gestión de la innovación instruccional curricular.

- Que entre las instituciones de educación superior haya al menos una de las universidades que ya estén acreditadas internacionalmente.

- Que la entidad coordinadora de la experiencia estuviera interesada en participar y apoyar el desarrollo de la investigación.

- Que en los grupos de estudio en los que participaran los implicados valoraran la formación en los programas profesionales de Contaduría Pública.

Con base en los criterios anteriores, se realizó la identificación para configurar el estudio de caso de las instituciones de educación superior en referencia: IUE, Unaula, a través de las cuales se identifican las categorías de referencia para este proceso investigativo y se usan como mediadoras de aprendizajes y de apoyo didáctico en procesos de armonización del currículo contable.

La segunda fase de la investigación fue dedicada especialmente a la recolección de la información que brindaron las distintas fuentes; las técnicas de recogida de datos utilizadas pasaron por los siguientes momentos:

a) la armonización de currículos, que simplifica lo propio de los currículos con sus unidades epistemológicas estructurantes,

b) la sistematización de asignaturas y de competencias por áreas y asignaturas, con sus respectivas mecánicas instrumentales (gestión de la innovación instrumental),

c) los consolidados de asignaturas y de contenidos, y d) los mapeos propios de competencias y de los conocimientos contables.

De igual forma, hicieron su aparición la observación participante, la entrevista a profundidad, el análisis documental y el diario de campo.

\section{Resultados}

\section{V.I. Un acercamiento a la armonización curricular de los programas contables}

Para acercarnos a este apartado recurrimos a definir la palabra currículo, a través de una voz autorizada como González (2008, p. 93), quien nos deja saber que currículo es una acción intencionada constitutiva de sentidos educativos. Como propuesta de acción académica y en construcción permanente, centra su atención en traducir la cultura de la humanidad y selecciona institucionalmente aquellos saberes pertinentes para ser objeto de enseñanza y de aprendizaje, con miras en la educación superior, de formar científicamente profesionales. 
Restrepo. D. y Cárdenas, R. Caracterización de la internacionalización del currículo contable...

Interesa seguir avanzando en las definiciones de nuestros conceptos centrales y recurrimos a la categoría Armonización, que deviene de armonía, que según la Real Academia de la Lengua (2019) "es hacer que concuerden dos cosas, para un mismo fin, el hacer que no discuerden o se rechacen dos o más partes de un todo, o dos o más cosas que deben concurrir al mismo fin". Haciendo una transposición del término al campo pedagógico y académico, la armonización curricular hace referencia a una propuesta que se plantea a partir de la búsqueda de sentidos educativos, concertar los elementos constitutivos de dos o más currículos, respetando su diversidad y sus componentes afines y no afines.

Relacionando las categorías que centran el interés de este proceso investigativo, armonización y currículo, y que configuran el propósito central de este texto, que es la armonización, se compararan los currículos de las IES en referencia a la Universidad Autónoma Latinoamericana, UNAULA, y a la Institución Universitaria de envigado, IUE, en la fase uno. En la segunda fase, que no es el objeto de este texto, nos compararemos con varias Instituciones de Educación Superior, que tienen los programas académicos de Contaduría Pública que se listan en el cuadro siguiente con el propósito de identificar los conocimientos y las competencias que en dichos programas se han desarrollado y que pudiesen homologarse.

Este cuadro nos relaciona cada uno de los programas de contaduría pública e instituciones de educación superior, IES, que se tendrán en cuenta para ser seleccionadas durante el desarrollo del proyecto en la fase dos de este proceso investigativo, porque para los propósitos de este escrito solo se da cuenta de un estudio de caso, IUE, y Unaula.

Cuadro 1: Programas de contaduría pública tenidos en cuenta

\begin{tabular}{ll}
\hline Programas de contaduría pública & \multicolumn{1}{c}{ Instituciones de Educación Superior } \\
\hline Contaduría Pública & Institución Universitaria de Envigado \\
Contaduría Pública & Universidad Autónoma Latinoamericana \\
Contaduría Pública & Universidad de Medellín \\
Contaduría Pública & Universidad EAFIT \\
Contaduría Pública & Universidad Autónoma de Bucaramanga \\
Contaduría Pública & Universidad Externado de Colombia \\
Contaduría Pública & Universidad Central \\
Contaduría Pública & Universidad de la Costa CUC \\
Contaduría Pública & Pontificia Universidad Javeriana, Cali \\
Licenciatura en Contaduría & Universidad Nacional Autónoma de México \\
Contador Público & Universidad de Buenos Aires \\
\hline
\end{tabular}

Fuente: Elaboración propia (2019) con base en la información compilada. 
Para ello se identificó en cada programa del caso de referencia (IUE, UNAULA), seleccionando sus constitutivos macro, meso y micro curricular, para establecer una comparación desde lo idéntico y no idéntico. Esta organización permitió diseñar una secuencia de mapas desde los elementos idénticos concentrados en competencias y conocimientos como un primer elemento valorativo de la relación entre la formación profesional y la formación por ciclos.

Para armonizar los currículos se desarrollaron los siguientes pasos con sus respectivos instrumentos, los cuales permiten la arquitectura propia de la armonización del currículo en el campo contable para las IES en referencia, IUE, UNAULA.

\section{V.II. Los retos de la sistematización de los currículos}

Para enfrentar la sistematización de los currículos en referencia, se corroboró a través de poner en uso el instrumento que describimos a continuación, y que tuvo como propósito ordenar la información macro curricular de los programas en referencia (IUE, Unaula), a partir de las siguientes categorías: nombre del programa, institución educativa, propósitos de formación, problemas de formación, perfil académico, perfil profesional, perfil ocupacional, objeto de estudio, objetivos, campos del conocimiento, nombre de las asignaturas y total de créditos.

Para dar cuenta de ello, en la configuración de este momento fue vital la participación de los profesores, en tanto fueron ellos quienes entregaron la información necesaria para cada una de las categorías mencionadas, por conocimiento y experticia en las labores propias del trabajo curricular desarrollado por ellos en la configuración y estudio de los planes de estudio en referencia.

\section{V.II.I. Mecánica Instrumental}

El propósito con el uso del instrumento en referencia deja saber de su mecánica de instrumentación para lograr sistematizar la información general de los currículos contables de las instituciones en referencia, en el que cada uno de los actores involucrados asumió cada una de las indicaciones que se explican a continuación.

Las indicaciones que se impartieron fueron que solo se diligenciaran por los profesores que hacen parte del colectivo de docencia y del área respectiva, ciencias básicas, profesionalización y humanidades.

$\mathrm{Si}$ alguno de los componentes no se encuentra en el programa, lo señalarán con las letras NE, No evidencia. 
Restrepo. D. y Cárdenas, R. Caracterización de la internacionalización del currículo contable...

Cuadro 2: Instrumento de componentes de los programas

\begin{tabular}{lc}
\hline \multicolumn{1}{c}{ Nombre del programa } & Institución educativa \\
\hline \multicolumn{1}{c}{ Contaduría Publica } & Unaula - IUE \\
\hline Propósitos de formación & \\
Problemas de formación & \\
Perfil académico & \\
Perfil profesional \\
Perfil ocupacional \\
Objeto de estudio \\
Objetivos \\
Campo del conocimiento \\
Nombre de la asignatura \\
Total de créditos
\end{tabular}

Fuente: Elaboración propia con base en los documentos de autoevaluación y acreditación institucionales.

En este apartado se permitió dar cuenta de los Proyectos Educativos del programa PEP de Contaduría Pública de las IES en referencia, de las cuales se recogieron elementos como: especificidad del programa, docencia, en cuanto a sus referentes nacionales e internacionales, aspectos curriculares, investigación, ciencia, tecnología e innovación, responsabilidad social institucional, en el que se considera el impacto del programa, extensión y proyección social, vinculación nacional e internacional, y aseguramiento de la calidad; en este último elemento se incluye la gestión del programa, resultados de autoevaluación y plan de mejoramiento.

De igual forma, la difusión y discusión del Proyecto Educativo desde los Programas, mostró que se realizó la socialización con los estudiantes, a través de la implementación de las cátedras propias de cada una de la IES en referencia, caso en la IUE, de "Pensamiento IUE", pensamiento latinoamericano y cátedra universitaria, Unaula, y en los procesos de inducción de los estudiantes. Para el caso de los docentes, la socialización se hizo a través de las reuniones de planeación de inicio de semestre, y a través de los procesos de sensibilización que acompañaron los procesos de Autoevaluación.

Por otro lado, interesó a este proceso identificar el propósito de formación de cada una de las IES del caso; veamos. El propósito de formación de Unaula reza:

El Programa busca a través de las prácticas pedagógicas de formación, que el estudiante aprenda a aprender, a ser, hacer, emprender y a convivir. Que asuma el preguntar como la exigencia básica de su aprendizaje, un preguntar inscrito en la dinámica, entre lo pensable y lo impensable, capaz de renovar los problemas y saberes codificados en la disciplina contable, la interrogación debe brindarle al estudiante la posibilidad de construir, a partir del diálogo y el intercambio de saberes. 
La formación en el aprendizaje busca que el futuro profesional, adquiera un compromiso permanente con el conocimiento. De suerte que esa relación sea el fundamento de las condiciones para su desarrollo personal, intelectual y social.

Buscamos no sólo formar con rigurosidad científica contadores públicos, sino desarrollar las competencias necesarias para formar seres humanos íntegros, coherentes, comprometidos con la sociedad y el entorno. $(2018$, p.87)

El propósito de formación de la Institución Universitaria de Envigado, dice así:

Facultar profesionales para asegurar la transparencia, utilidad y confiabilidad de la información, así como la generación de confianza pública a través de procesos relacionados con la medición del desempeño contable y financiero de las organizaciones, su interpretación y posibles implicaciones, conforme a las normas nacionales e internacionales. (2017, p. 13)

El perfil del contador Unaulista deja saber:

El Contador Público de UNAULA será un profesional integral con amplios conocimientos científico-técnicos, con formación fundamentada en el pensamiento crítico y en el espíritu investigativo, consciente del contexto, la interdisciplinariedad, la ciencia y la complementariedad de las prácticas sociales, en su tarea de satisfacer necesidades y contribuir al mejoramiento del bienestar y calidad de vida de la población.

Será una persona con habilidades académicas, profesionales y sociales, capaz de participar en la solución de conflictos a través de procesos dialógicos; de emitir juicios profesionales sobre la continuidad de las organizaciones de diverso orden, soportados en el diseño, la implementación y la administración de sistemas de información contable y de control, atendiendo las necesidades de información de los agentes que participan de la vida económica de la nación, orientando su labor a la protección del interés público, en los sectores público, privado y social y en los ámbitos local, nacional e internacional.

Consciente que su saber se inscribe en el campo de las ciencias económicas, reconoce su compromiso con la tarea de informar y controlar los procesos de generación, distribución y redistribución de la riqueza, además del uso productivo y responsable de los recursos.

Sus actuaciones estarán regidas por los más altos principios éticos y valores morales, como constructor de confianza pública. Será un ciudadano respetuoso de la diferencia y promotor de niveles de convivencia y de un orden social justo, equitativo e incluyente.

Adicionalmente, será sensible con el entorno social y natural, defensor del principio de igualdad biocéntrica como condición necesaria para la preservación del planeta y de la especie humana. (2018, p. 89)

El perfil Profesional de la IUE, deja saber que: 
Restrepo. D. y Cárdenas, R. Caracterización de la internacionalización del currículo contable...

El egresado del Programa de Contaduría Pública tendrá la capacidad de desempañarse en entornos disruptivos, imprevisibles y complejos que son propios de contextos económicos y tecnológicos globalizados, con el fin de solucionar desde sus competencias técnicas y científicas problemas propios a la construcción, control y certificación de información, para comunicarla de forma efectiva como conocimiento útil en el proceso de desarrollo de nuevas oportunidades que se presentan tanto a las personas como a los entes económicos, en especial en el campo tributario y financiero. (2017, p. 15)

El perfil Ocupacional de la IUE, Dice:

El Contador Público egresado de la IUE se podrá desempeñar como:

- Contador de empresas privadas y públicas.

- Jefe de costos, presupuesto e impuestos.

- Jefe de área financiera.

- Asesor tributario y contable.

- Auditor.

- Analista financiero.

- Revisor fiscal.

- Contralor.

- Tesorero.

- Decano facultades de Contaduría Pública.

- Gestor de su propia empresa. (2017, p. 16)

Cada una de las categorías anteriores permite configurar los elementos centrales de la estructura macro curricular que da pie para la orientación de la armonización curricular que en estas páginas se configura.

\section{V.III. La sistematización de asignaturas}

La sistematización de asignaturas se puede corroborar a través del instrumento en uso para ello, que tuvo como propósito ordenar la información del meso currículo a través de la identificación de las diferentes asignaturas con relación al número de créditos, con sus respectivas horas de trabajo directo e independiente, los problemas, objetivos y contenidos básicos.

\section{V.III.I. Mecánica Instrumental}

El propósito de este instrumento es caracterizar las diferentes asignaturas de acuerdo con el número de créditos y su correspondiente distribución; así como identificar los problemas, objetivos y contenidos básicos.

Las Indicaciones que se impartieron al grupo de profesores dejó saber que este instructivo debía ser diligenciado por cada uno de los profesores que pertenecen al área respectiva de formación de la siguiente forma: profesores 
del área básica diligencian la información de las materias específicas que dictan en dicha área; igual los de profesionalización y de humanidades.

1. Nombre de la asignatura

2. Número de créditos

3. Horas de trabajo directo

4. Horas de trabajo independiente

5. Problema

6. Objetivos

7. Contenidos básicos

En este apartado se permite dar cuenta que la sistematización de las asignaturas van de acuerdo con el modelo pedagógico de cada institución, así como de las estrategias didácticas que adopta el programa de Contaduría Pública respectivo y que van directamente dando cuenta del trabajo académico presencial, a partir de que en los encuentros presenciales el programa asume la propuesta del modelo pedagógico dialógico con estrategias, donde más allá de lo magistral, se puedan suscitar espacios para la intersubjetividad y la discusión contenida en el marco de lo propuesto en cada curso.

Para consolidar la propuesta pedagógica dialógica y diversificar los encuentros en el aula, se utilizan estrategias como las que se enuncian a continuación: trabajos en grupos colaborativos, defensa de posiciones entre grupos o pares de forma individual, solución de problemas hipotéticos y reales, cursos tipo seminario, encuentros entre teorías, encuentros interdisciplinarios, desarrollo de proyectos, lecturas previas y discusiones, conferencias magistrales con un segundo momento para la discusión, y otras prácticas que permiten el encuentro en función de la disciplina de interés.

De igual forma, se permite dar cuenta del trabajo académico independiente; este se consolida en productos en cada asignatura, que se van plasmando a través de guías, protocolos, relatorías, trabajo individual y de grupo, portafolios y tutorías, entre otros. En igual sentido, nos permite analizar cómo el docente flexibiliza el acompañamiento directo al estudiante, al trabajo independiente que éste realiza apoyado desde una pedagogía dialógica y analítica.

Finalmente, se deja saber de actividades académicas complementarias extracurriculares, a través de las cuales se cuenta con los espacios para enriquecer y complementar la formación académica de los estudiantes y egresados, tales como: debates, capacitaciones, cine foros y seminarios con invitados nacionales e internacionales, participación en semilleros de investigación, donde se abordan temáticas que merecen su atención al ser importantes, pero no son un requerimiento dentro del currículo de formación de pregrado en Contaduría Pública en las instituciones de referencia. 


\section{V.IV. La sistematización de competencias}

La sistematización de competencias se puede corroborar a través del instrumento en uso para ello, que tuvo como propósito identificar las diferentes competencias de las asignaturas respectivas, luego de la lectura de los micro currículos. Para ello fue necesario listar las competencias tal como estuviesen clasificadas en cada asignatura para luego reclasificarlas. Si las asignaturas no tenían sus competencias explícitas, se listaban los verbos con los cuales se iniciaba cada uno de los objetivos de las asignaturas, siempre y cuando dichos verbos representaran una capacidad a desarrollar por los estudiantes.

La clasificación de las competencias fue la siguiente: competencias generales, básicas, específicas, transversales, científicas, profesionales, laborales, socio afectivas, comunicativas, propositivas, interpretativas, argumentativas y otras, si las hubiese.

\section{V.IV.I. Mecánica Instrumental}

El propósito de este instrumento es identificar las competencias en cada uno de los programas en referencia.

Las Indicaciones que se impartieron, orientaban en el cómo diligenciar el instructivo haciendo el listado de competencias según como estén clasificadas en su programa. Si los programas no tienen explícitas las competencias, hacer un listado con los verbos con los cuales se inician cada uno de los objetivos del programa (Carta descriptiva o Syllabus) o de los micro currículos.

1. Institución

2. Competencias

2.1 Generales

2.2 Básicas

2.3 Específicas

2.4 Transversales

2.5 Científicas

2.6 Profesionales

2.7 Laborales

1. Institución

2. Competencias

2.1 Socio afectiva

2.2 Comunicativas

2.3 Propositivas

2.4 Interpretativas

2.5 Argumentativas otras 


\section{V.V. Consolidado de competencias por áreas y asignaturas}

El consolidado de competencias por áreas y asignaturas, se puede corroborar a través del instrumento en uso para ello; tuvo como propósito recopilar la información de la sistematización de competencias a través del criterio de identidad y no identidad, pues la armonización curricular debe constituirse desde los elementos comunes a todos los currículos analizados, para poder establecer los homogéneos que serán la base de la prueba de evaluación por competencias en el ciclo respectivo.

Para elaborar el consolidado fue necesario registrar el nombre de las instituciones que ofrecen el programa o programas afines, identificando las áreas comunes en programas afines, así como también las asignaturas comunes en el área respectiva, para identificar, desde allí, las competencias comunes y diferentes. Obviamente, al identificar lo común se encuentra lo diferente, lo cual no se tuvo en cuenta para el diseño de la prueba.

\section{V.V.I. Mecánica Instrumental}

El propósito de este instrumento es consolidar las competencias por áreas y asignaturas

Las Indicaciones para hacer uso de este instrumento, deja saber que se debe diligenciar de la siguiente manera: se reunirán los profesores por áreas de formación, con el fin de identificar qué asignaturas de las áreas son comunes y en estas asignaturas, qué competencias son comunes o diferentes. El instrumento consta de seis columnas:

- Primera columna. Se refiere a la denominación de las instituciones que ofrecen el programa. Se sugiere que determinen las instituciones dónde corresponden los programas.

- Segunda columna. Se deben ubicar los programas con nominación igual.

- Tercera columna. Corresponder a la identificación de las áreas comunes por programa común. Esta información está concatenada con las anteriores columnas. Donde haya un área de énfasis, profundización o línea diferente, se ubica al final de la columna tres, como última área analizada de cada programa y se específica que es un área disímil de $\mathrm{X}$ programa y o de qué institución.

- Cuarta columna. Asignaturas comunes. En ella se requiere ubicar las asignaturas afines con el área y el programa común. Igual que con la anterior columna, al final se ubicarán las asignaturas disímiles de las áreas comunes, haciendo los respectivos comentarios. En esta columna también se listan las asignaturas disímiles de las áreas que no lo son.

- Quinta columna. Corresponde a los numerales referidos a las nominaciones de cada una de las competencias listadas en el instrumento respectivo de las competencias, y referenciadas anteriormente. En esta columna se 
Restrepo. D. y Cárdenas, R. Caracterización de la internacionalización del currículo contable...

ubican las competencias comunes que corresponden con el ítem. Recuerde que la información está concatenada entre columnas.

- Sexta columna. Referida a las competencias diferentes. Se diligencia de la misma manera que la columna cinco, solo que en este caso son las competencias disímiles.

\section{V.VI. Consolidado de asignaturas}

El consolidado de asignaturas se puede corroborar a través del instrumento en uso para ello; se tuvo como propósito recopilar, con el criterio de identidad y no identidad, tanto las asignaturas como el trabajo en créditos de cada una de ellas y las horas de trabajo directo e independiente.

\section{V.VI.I. Mecánica Instrumental}

El propósito de este instrumento es consolidar las asignaturas de los programas comunes y diferentes en relación con los créditos académicos.

Las indicaciones que nos guían en este apartado se centran en una serie de columnas que nos grafican la búsqueda propuesta, veamos.

- Primera columna. Se registran las instituciones que ofrecen programas con la misma nominación.

- Segunda columna. Se ubican los programas que tienen la misma nominación en correspondencia con la columna uno.

- Tercera columna. Se escribe el área común a cada programa y al final el área diferente al programa.

- Cuarta columna. Se listan las asignaturas comunes de cada programa en relación con la columna anterior.

- Quinta columna. Se ubican el número de créditos comunes con relación a cada asignatura de la columna tres. Al final de esta misma columna se van ubicando los créditos disímiles en correspondencia con las asignaturas de la columna anterior.

- Sexta columna. Se ubican las horas de trabajo directo, en correspondencia con el número de créditos listados en la columna anterior.

- Séptima columna. Igual que en la columna seis, se listan las horas de trabajo independiente, en correspondencia con las columnas seis, cinco y cuatro.

- Octava columna. Se listan las asignaturas disímiles, buscando que se correspondan con el área de la comuna tres y el programa de la columna dos. Las columnas nueve, diez y once, se diligencian con la misma lógica de las columnas cinco, seis y siete. 
Cuadro 3: Información sobre UNAULA

\begin{tabular}{|c|c|c|}
\hline INSTITUCIÓN & UNAULA & IUE \\
\hline Programas & Contaduría publica & Contaduría pública \\
\hline Áreas & $\begin{array}{l}\text { 1. Básicas, 2. Profesional, 3. Socio } \\
\text { humanística, } 4 \text {. De formación científica } \\
\text { e investigación, } 5 \text {. Electiva de } \\
\text { profundización. }\end{array}$ & $\begin{array}{l}\text { 1. Básica, 2. Profesional } 3 . \\
\text { Socio humanística. }\end{array}$ \\
\hline $\begin{array}{l}\text { Áreas y } \\
\text { componentes }\end{array}$ & $\begin{array}{l}\text { Básicas: } \\
\text { De matemáticas y estadísticas, de } \\
\text { economía, de ciencias jurídicas. } \\
\text { Profesional: } \\
\text { De ciencias contables: contabilidad } \\
\text { financiera, costos y presupuestos, } \\
\text { finanzas, control. } \\
\text { De formación organizacional: en } \\
\text { sistemas de información, teoría contable } \\
\text { y regulación } \\
\text { Socio humanística } \\
\text { De formación científica y en investigación } \\
\text { Electiva de profundización }\end{array}$ & $\begin{array}{l}\text { Básicas: } \\
\text { Matemáticas, estadísticas, } \\
\text { economía, ciencias } \\
\text { jurídicas y administrativas } \\
\text { Profesional: } \\
\text { Ciencias contables y } \\
\text { financieras, formación } \\
\text { organizacional, } \\
\text { información, regulación, } \\
\text { proyecto de grado. } \\
\text { Socio humanísticas: } \\
\text { interdisciplinariedad. }\end{array}$ \\
\hline $\begin{array}{l}\text { Asignaturas } \\
\text { comunes }\end{array}$ & \multicolumn{2}{|c|}{ Las contables, las auditorias, las tributarias y financieras } \\
\hline Créditos & $\begin{array}{l}\text { Básica: Unaula (46) } 27.2 \% \\
\text { Profesional: Unaula (88) 52\% } \\
\text { Socio humanística: Unaula (16) 9.5\% }\end{array}$ & $\begin{array}{l}\text { Básica: IUE (33) 19.5\% } \\
\text { Profesional: IUE (103) 61\% } \\
\text { Socio humanística: IUE (33) } \\
19.5 \%\end{array}$ \\
\hline $\begin{array}{l}\text { Horas de trabajo } \\
\text { directo }\end{array}$ & 32 & 32 \\
\hline $\begin{array}{l}\text { Horas de trabajo } \\
\text { independientes }\end{array}$ & 48 & 48 \\
\hline $\begin{array}{l}\text { Asignaturas } \\
\text { diferentes }\end{array}$ & 4 & 5 \\
\hline
\end{tabular}

Fuente: Elaboración propia con base en los documentos de autoevaluación y acreditación institucionales.

La información compilada en el cuadro anterior, nos permite sostener lo propio de cada área de formación así:

\section{Área de Formación Básica:}

- Componente Matemática: Apoya la estructura del pensamiento lógico racional del estudiante y ayuda a las comprensiones de los métodos deductivos, inductivos e inferenciales de los fenómenos propios de la información contable. 
Restrepo. D. y Cárdenas, R. Caracterización de la internacionalización del currículo contable...

- Componente Estadística: La información de las empresas tiene un comportamiento a través del tiempo, el cual es analizado por medio de modelos estadísticos, para una mejor toma de decisiones, a partir de tendencias encontradas según los estudios.

- Componente Economía: Este componente tiene como finalidad formar al estudiante con una visión nacional e internacional en el ámbito económico, mirando la influencia que tienen las variables económicas en los sectores productivos, lo cual ayuda para la planeación organizacional, para el diseño e implementación de modelos de trabajo en los ámbitos de la profesión contable.

- Componente Ciencias Jurídicas y Administrativas: La contaduría Pública en sus diferentes ámbitos de desempeño está regida por una amplia normatividad como lo es el derecho tributario, laboral y comercial, además de su propia regulación, como es lo referido a las normas internacionales de contabilidad y a las normas internacionales de auditoría o de aseguramiento de la información financiera.

\section{Área de Formación Profesional:}

- Componente Ciencias Contables y Financieras: Más que un sistema de información que presenta los estados financieros de los distintos entes económicos para el análisis y toma de decisiones.

- Componente Formación Organizacional: Otorga habilidades y competencias para la interrelación en campos organizacionales complejos e imprevisibles, con el ánimo de analizar la información propia de las necesidades y requerimientos de los grupos de interés.

- Componente Información: El desarrollo de la tecnología, tanto en máquinas como en sistemas, ha cambiado el desempeño de la contaduría, llevando en primer lugar al manejo de sistemas contables y de componentes de ofimática, que mejoran el manejo y calidad de la información para la toma de decisiones; además, se hace necesario la interpretación de la información.

- Componente regulación: En lo contable se debe asumir las leyes, decretos, reglamentos y regulaciones, para poner en práctica el saber contable.

Proyecto de grado: Es el estudio sistemático de determinada área de la Contaduría, el cual implica un proceso de observación, exploración, descripción, interpretación y explicación, desarrollado durante el proceso de formación.

Área de Formación socio-humanística: Saberes y prácticas para la formación integral del estudiante, en virtud de potenciar el proyecto de formación y de vida de cada uno de los involucrados en el proceso. 


\section{V.VII. Consolidado de contenidos}

El consolidado de los contenidos se logra a partir de la identificación de las asignaturas idénticas a través de las cuales se identifican y se seleccionan sus contenidos; se puede corroborar a través del instrumento en uso para ello; tuvo como objetivo recopilar los conocimientos de las asignaturas comunes e identificar cuáles contenidos son similares y cuáles completamente diferentes. Una vez más este consolidado se hizo teniendo en cuenta el criterio de armonizar los currículos desde lo idéntico para la selección de los contenidos que permitieran diseñar la prueba en referencia.

\section{V.VII.I. Mecánica Instrumental}

El propósito de este instrumento es consolidar los contenidos de las asignaturas de los programas comunes, y las indicaciones que se comparten para configurar su sistematización se da a través de las siguientes columnas.

- Primera columna. Se registran las instituciones que ofrecen programas con la misma nominación.

- Segunda columna. Se ubican los programas que tienen la misma nominación en correspondencia con la columna uno.

- Tercera columna. Ubicar el área común a cada programa y al final el área diferente al programa.

- Cuarta columna. Se listan las asignaturas comunes de cada programa en relación con la columna anterior.

- Quinta columna. Se listan los contenidos básicos comunes de cada una de las asignaturas. Recuerde que la información está concatenada entre las columnas.

- Sexta columna. Se listan los contenidos básicos diferentes de cada una de las asignaturas.

Cuadro 4: Contenido de las asignaturas por programas comunes

\begin{tabular}{lcll}
\hline Institución & Asignaturas & Contenidos básicos comunes & Contenidos básicos diferentes \\
\hline Unaula & 62 & Las contables & Cátedra latinoamericana \\
IUE & 63 & Pensamiento político & Pensamiento IUE, \\
\hline
\end{tabular}

Fuente: Elaboración propia con base en los documentos de autoevaluación y acreditación institucionales.

\section{V.VIII. Mapeo de competencias y conocimientos contables}

Erigir el mapeo como estrategia central de este proceso de investigación centrado en la armonización del currículo contable en las instituciones de educación superior de referencia, en tanto representación gráfica de las competencias y de los conocimientos contables básicos, es una estrategia sintetizadora de la información que se va construyendo a partir de las inferencias de cada uno de los momentos bosquejados. 
Restrepo. D. y Cárdenas, R. Caracterización de la internacionalización del currículo contable...

El primer momento, se elabora a partir de la lectura de la armonización de los currículos contables. Este mapa permitirá apreciar, en forma de síntesis, una primera comparación entre las áreas, conocimientos y competencias que comparten los planes de estudio en referencia.

Cuadro 5: Armonización de los currículos contables

\begin{tabular}{|c|c|c|}
\hline AREAS & $\begin{array}{c}\text { CONOCIMIENTOS } \\
\text { Componentes curriculares y créditos. }\end{array}$ & COMPETENCIAS \\
\hline $\begin{array}{l}\text { Básica } \\
\text { Unaula (46) } 27.2 \%\end{array}$ & $\begin{array}{l}\text { Unaula: Matemáticas (13) y estadística (3), } \\
\text { economía (18), ciencias Jurídicas (12). }\end{array}$ & \multirow{2}{*}{$\begin{array}{l}\text { Analizar (comprender), demostrar, } \\
\text { reconocer, deducir, elaborar, } \\
\text { describir, aplicar, obtener, definir, } \\
\text { diferenciar, identificar, resolver, } \\
\text { determinar, reconocer. }\end{array}$} \\
\hline IUE (33) $19.5 \%$ & $\begin{array}{l}\text { IUE: Matemáticas (9), estadística (6), economía } \\
\text { (9), ciencias Jurídicas y administrativas (9). }\end{array}$ & \\
\hline $\begin{array}{l}\text { Profesional: } \\
\text { Unaula (88) } 52 \%\end{array}$ & $\begin{array}{l}\text { Unaula: Ciencias contables (65), Formación } \\
\text { organizacional (10), Sistemas de información, } \\
\text { Teoría contable (10) y regulación (3). }\end{array}$ & \multirow{2}{*}{$\begin{array}{l}\text { Aplicar (diagnosticar) administrar, } \\
\text { calcular, controlar, identificar, } \\
\text { interpretar, medir, mejorar, } \\
\text { registrar, resolver, transferir. }\end{array}$} \\
\hline IUE (103) $61 \%$ & $\begin{array}{l}\text { IUE: Ciencias contables y financieras (63), } \\
\text { formación organizacional (9), información (9), } \\
\text { regulación (9), proyecto de grado (13). }\end{array}$ & \\
\hline & & \multirow{3}{*}{$\begin{array}{l}\text { Interpretar (proponer) } \\
\text { caracterizar, comprobar, describir, } \\
\text { observar, verificar, visualizar, } \\
\text { aplicar. }\end{array}$} \\
\hline Unaula (16) 9.5\% & $\begin{array}{l}\text { Habilidades Comunicativas, Emprendimiento, } \\
\text { Seminario Humanístico I y II, Cátedra } \\
\text { Universitaria, Historia de América Latina, } \\
\text { Comprensión Lectora de Inglés I y II }\end{array}$ & \\
\hline & IUE: interdisciplinariedad (33). & \\
\hline $\begin{array}{l}\text { Electiva de } \\
\text { profundización }\end{array}$ & $\begin{array}{l}\text { Unaula: Electiva de profundización (40): } \\
\text { Revisoría Fiscal, Finanzas, Emprendimiento } \\
\text { empresarial, Gestión tributar, Gerencia } \\
\text { estratégica de costos. } \\
\text { De formación científica y en investigación (9): } \\
\text { Seminario de Investigación I y II, Opción de } \\
\text { Grado I y II. }\end{array}$ & $\begin{array}{l}\text { Clasificar, comparar, demostrar, } \\
\text { explicar, identificar, recopilar, } \\
\text { ordenar, programar, relacionar, } \\
\text { simplificar, utilizar, sintetizar. }\end{array}$ \\
\hline & $\begin{array}{l}\text { IUE: electiva, optativa, seminario de } \\
\text { profundización }\end{array}$ & Obtener, diferenciar, caracterizar \\
\hline \multicolumn{3}{|c|}{ Fuente: Elaboración propia con base en los documentos de autoevaluación 2017} \\
\hline - El compor & $\begin{array}{l}\text { n reflejada en el cuadro anterior s } \\
\text { respectivos, así: }\end{array}$ & $\begin{array}{l}\text { puede analizar a partir de } \\
\text { uciones deja saber que se } \\
\text { ivos, inductivo e inferencial } \\
\text { ivosal del estudiante y }\end{array}$ \\
\hline
\end{tabular}


- El componente estadístico muestra que la información de las empresas tiene un comportamiento a través del tiempo, el cual es analizado por medio de modelos estadísticos que ayudan a una mejor toma de decisiones, a partir de tendencias encontradas en los estudios recientes.

- El componente de economía deja saber la finalidad de formar al estudiante con una visión nacional e internacional en el ámbito económico, mirando la influencia que tienen las variables económicas en los sectores productivos, lo cual ayuda para la planeación organizacional, el diseño e implementación de modelos de trabajo en los ámbitos de la profesión contable.

- $\quad$ El componente de ciencias jurídicas y administrativas deja saber los diferentes ámbitos de desempeño, los cuales están regidos por una amplia normatividad como lo es el derecho tributario, laboral y comercial; además de su propia regulación que es referida a normas internacionales contables y a normas internacionales de auditoría o de aseguramiento de la información financiera.

El segundo momento, agrupa en un cuadro que compila las áreas y sus correspondientes asignaturas, para mostrar cómo aumentan o disminuyen las frecuencias absolutas con relación al número de preguntas generadoras de búsquedas y de problemas que configuran el plan de estudios, así como los conocimientos y sus respectivas competencias.

Cuadro 6: Variación de preguntas y problemas del plan de estudios

\begin{tabular}{|c|c|c|c|c|c|c|}
\hline 苞 & Asignaturas & $\begin{array}{l}\text { Número de } \\
\text { problemas }\end{array}$ & $\begin{array}{l}\text { Numero de } \\
\text { preguntas }\end{array}$ & Competencias & $\mathrm{Fr}$ & Conocimientos \\
\hline & $\begin{array}{l}\text { Unaula } 16 \\
\text { Matemáticas } \\
\text { Estadística } \\
\text { Economía Ciencias Jurídicas }\end{array}$ & 3 & 2 & $\begin{array}{l}\text { Interpretar } \\
\text { Relacionar } \\
\text { Modelar }\end{array}$ & * & \\
\hline 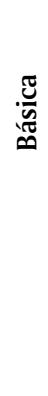 & $\begin{array}{l}\text { IUE: } 11 \text { Matemáticas } \\
\text { operativas, microeconomía, } \\
\text { calculo, macroeconomía, } \\
\text { administración general, } \\
\text { Estadística descriptiva, } \\
\text { Economía nacional e } \\
\text { internacional, legislación } \\
\text { comercial y societaria, } \\
\text { estadística inferencial, } \\
\text { legislación laboral, } \\
\text { investigación de operaciones. }\end{array}$ & 3 & 2 & $\begin{array}{l}\text { Simbolizar } \\
\text { Deducir } \\
\text { generalizar }\end{array}$ & & 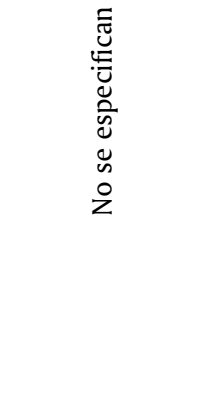 \\
\hline
\end{tabular}


Restrepo. D. y Cárdenas, R. Caracterización de la internacionalización del currículo contable...

\begin{tabular}{|c|c|c|c|c|c|c|}
\hline 离 & Asignaturas & $\begin{array}{l}\text { Número de } \\
\text { problemas }\end{array}$ & $\begin{array}{l}\text { Numero de } \\
\text { preguntas }\end{array}$ & Competencias & $\mathrm{Fr}$ & Conocimientos \\
\hline 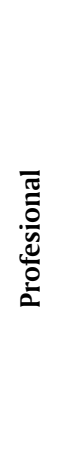 & $\begin{array}{l}\text { Unaula } 32 \\
\text { Ciencias contables Formación } \\
\text { organizacional Sistemas de } \\
\text { información, Teoría contable y } \\
\text { regulación. } \\
\text { IUE: } 103 \\
\text { Ciencias contables y } \\
\text { financieras, formación } \\
\text { organizacional, información, } \\
\text { regulación, proyecto de grado. }\end{array}$ & 3 & 6 & $\begin{array}{l}\text { Modelar } \\
\text { Interpretar } \\
\text { formalizar }\end{array}$ & $*$ & 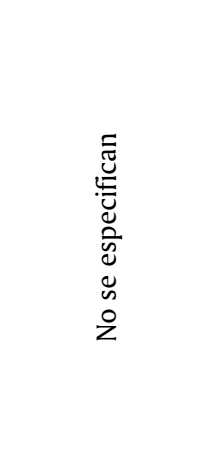 \\
\hline 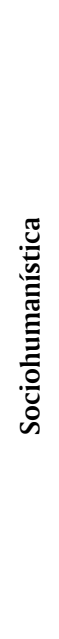 & $\begin{array}{l}\text { Unaula } 14 \\
\text { Sociohumanística: } \\
\text { Habilidades Comunicativas, } \\
\text { Emprendimiento, Seminario } \\
\text { Humanístico I y II, Cátedra } \\
\text { Universitaria, Historia de } \\
\text { América Latina, Comprensión } \\
\text { Lectora de Inglés I y II } \\
\text { IUE:15 } \\
\text { Interdisciplinariedad } \\
\text { Taller de lenguaje, procesos } \\
\text { culturales, pensamiento } \\
\text { IUE, procesos políticos, } \\
\text { metodología de la } \\
\text { investigación, electiva, inglés I } \\
\text { y II, ética profesional, optativa, } \\
\text { proyecto de investigación. }\end{array}$ & 5 & 5 & $\begin{array}{l}\text { Comprender } \\
\text { Analizar }\end{array}$ & * & 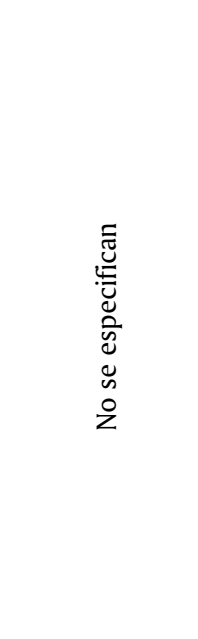 \\
\hline
\end{tabular}

Fuente: Elaboración propia con base en los documentos de autoevaluación 2017.

Para ir configurando lo bosquejado en líneas precedentes, se hace necesario ubicar los propósitos que se tienen; veamos:

Propósitos de formación: asegurar transparencia utilidad y confiabilidad de la información, así como generar confianza pública a través de procesos relacionados con la medición del desempeño contable y financiero de las organizaciones, su interpretación e implicaciones que se tienen en función de las normas nacionales e internacionales. 
Cuadro 7: Propósitos de formación

Propósito de formación

Unaula
Propósito de formación

IUE
El Programa busca a través de las prácticas pedagógicas de formación, que el estudiante aprenda a ser, hacer, emprender y a convivir. Que asuma el preguntar como la exigencia básica de su aprendizaje; un preguntar inscrito en la dinámica, entre lo pensable y lo impensable, capaz de renovar los problemas y saberes codificados en la disciplina contable; la interrogación debe brindarle al estudiante la posibilidad de construir, a partir del diálogo y el intercambio de saberes.

La formación en el aprendizaje busca que el futuro profesional adquiera un compromiso permanente con el conocimiento. De suerte que esa relación sea el fundamento de las condiciones para su desarrollo personal, intelectual y social.
Facultar profesionales para asegurar la transparencia, utilidad y confiabilidad de la información, así como la generación de confianza pública a través de procesos relacionados con la medición del desempeño contable y financiero de las organizaciones, su interpretación y posibles implicaciones, conforme a las normas nacionales e internacionales.

Fuente: Elaboración propia con base en los documentos de autoevaluación 2017.

De otro lado, se hace necesario identificar los campos de las competencias, que nos reflejan lo siguiente: Competencias genéricas y específicas: que se generan para los planes de estudio retoman del proyecto Tuning para América Latina en su informe de educación superior; reflexiones y perspectivas establece que: dominar los lenguajes, las técnicas y las prácticas contables en el marco de principios y normas generalmente aceptadas, así como usar los sistemas de información como soporte para el ejercicio profesional contable atendiendo criterios de utilidad, pertinencia y claridad; al igual comprender el contexto social, empresarial, legal, político e institucional, en el que se inscribe el ejercicio profesional contable, para dar trámite pertinente a su desempeño; y finalmente apropiar las teorías relacionadas con lo contable, conforme a las tendencias internacionales y al desempeño global de las organizaciones.

En este momento, se sintetiza la información recopilada en el momento anterior, es decir, se consolida la información de las competencias desde las disciplinas o áreas que le son propias, su sumatoria y porcentaje. Con ello se visualizan más claramente las competencias por su porcentaje de repetición y el grado de importancia que tengan en los procesos de formación de los estudiantes de contaduría pública, como se configura en la gráfica siguiente.

Cuadro 8: Áreas de conocimiento y competencias sugeridas

\begin{tabular}{ll}
\hline \multicolumn{1}{c}{ AREAS } & \multicolumn{1}{c}{ COMPETENCIAS SUGERIDAS } \\
\hline Básica & Deducir, demostrar, describir. \\
Profesional & Aplicar, obtener, definir, diferenciar. \\
Sociohumanística & Resolver, reconocer, determinar. \\
\hline
\end{tabular}

Fuente: Elaboración propia con base en los documentos de autoevaluación 2017. 
Restrepo. D. y Cárdenas, R. Caracterización de la internacionalización del currículo contable...

El momento que se configura aquí, muestra la oportunidad de comparar o relacionar con lo que aporta el proyecto Alfa Tuning América Latina, que busca "afinar" las estructuras educativas de América Latina iniciando un debate cuya meta es identificar e intercambiar información y mejorar la colaboración entre las instituciones de educación superior para el desarrollo de la calidad, efectividad y transparencia, y las ubica como genéricas, básicas, específicas y transversales, veamos.

Cuadro 9: Recopilación

\begin{tabular}{|c|c|c|c|c|c|}
\hline Programa & Disciplina/áreas & Competencias & Sumatoria & $\%$ & observaciones \\
\hline Unaula & $\begin{array}{l}\text { Básica } \\
\text { Profesional } \\
\text { Socio humanística }\end{array}$ & $\begin{array}{l}\text { Analizar, interpretar, deducir } \\
\text { Relacionar, concluir, abstraer } \\
\text { Generalizar, interpretar, } \\
\text { comprender }\end{array}$ & No aplica & & $\begin{array}{l}\text { Genérica } \\
\text { Transferir } \\
\text { conocimiento } \\
\text { deducir }\end{array}$ \\
\hline IUE & $\begin{array}{l}\text { Básica } \\
\text { Profesional } \\
\text { Socio humanística }\end{array}$ & $\begin{array}{l}\text { Aplicar, diferenciar, identificar } \\
\text { Relacionar, seleccionar }\end{array}$ & No aplica & & $\begin{array}{l}\text { Deducir } \\
\text { Transferencia } \\
\text { clasificar }\end{array}$ \\
\hline
\end{tabular}

Fuente: Elaboración propia con base en los documentos de autoevaluación 2017.

Con base en lo descrito en la gráfica anterior, se configura un nuevo momento; muestra este, la síntesis del proceso, y se apoya tanto en los momentos anteriores como en fuentes reconocidas: Redfacont, red de facultades de contaduría pública, y el proyecto Alfa Tuning América Latina, y en la socialización con los profesores provenientes de los programas en referencia.

Cuadro 10: Síntesis del proceso

\begin{tabular}{ccl}
\hline Competencias genéricas & Competencias básicas & \multicolumn{1}{c}{ Competencias específicas } \\
\hline Comprender, analizar e interpretar. & Formalizar, deducir, clasificar. & $\begin{array}{l}\text { Controlar procesos contables, } \\
\text { administrativos y financieros. }\end{array}$ \\
\hline
\end{tabular}

Fuente: Elaboración propia con base en los documentos de autoevaluación 2017.

\section{VI.Conclusión}

Se puede llegar a concluir cuando se visualiza el mapeo de las competencias y de los conocimientos que se bosquejaron en los pasos precedentes, que no se encuentran definidas las competencias de formación y que no se evalúan para determinar la eficacia de las mismas. Se reitera que solamente se formulan los objetivos que, en su gran mayoría, se caracterizan por estar centrados en dar la información al estudiante y no en los procesos de aprendizaje.

En relación con los problemas de formación, estos solo se hacen evidentes en el programa de la IUE, los demás no los describen. A su vez, desde el análisis de los contenidos se evidencian algunos aspectos coincidentes en el segundo mapeo, que en las líneas precedentes se bosquejaron. También se vislumbró que 
los dos programas trabajan con créditos académicos, aunque en las asignaturas comunes de los programas no se guarde la misma correspondencia en horas de trabajo académico. Es de anotar que en los programas seleccionados tanto en Unaula, como en la IUE, los créditos no tienen en cuenta las horas de trabajo independiente de los estudiantes.

Otra conclusión llama la atención en que algunas asignaturas de los programas en referencia tienen la misma denominación, aunque el nombre de la facultad difiere, pero carecen de homogeneidad en el tratamiento de los contenidos y en el manejo de sus créditos. Otras asignaturas cuentan con la misma denominación y contenidos, pero difieren en el número de créditos que se asignan.

En términos generales, a excepción de los programas en referencia, no se vislumbran cambios significativos en la malla curricular, dando la sensación de que se privilegia una concepción curricular tradicional. Asimismo, es importante señalar que los currículos de las materias de los planes de estudio en referencia no requieren de una transformación curricular para recibir a los jóvenes que ingresan por primera vez a su plan de formación personal, profesional y laboral, puesto que ellos dan cuenta de los conocimientos y competencias que se identificaron con la armonización y el mapeo que se configuró en los ítems respectivos a lo largo del trabajo descrito en estas páginas y que permiten aseverar que se enfatiza en el desarrollo de competencias genéricas, tal como se evidenciaron a través de comprender, analizar interpretar y diseñar en el campo contable al cual el estudiante es admitido, para cualificar la capacidad de controlar procesos contables, administrativos y financieros, en los que el estudiante busca perfeccionarse en el tiempo que dura su formación.

Finalmente, se puede aseverar que es necesario constituir un grupo de apoyo que entregue tutorías, que bien se pueden configurar a través de los distintos consultorios contables, como fases de la proyección social de los mismos y como una gran oportunidad para generar procesos de inclusión y de permanencia al interior de las instituciones que estructuraron el caso de estudio, para viabilizar la formación por competencias como urgente, y como actitud manifiesta de la capacidad que tiene el ser humano, en tanto es ser en potencia, para resolver problemas con el uso de conocimiento que se adquiere metódicamente.

\section{Referencias bibliográficas}

Aponte, C. (2016). Internacionalización académica; convenios, educación virtual, estudios en el exterior, pares académicos y redes. Chía: Universidad de la Sabana.

Bobbitt, F. (2008). Social Efficiency Movement. Retrieved November 4, from a hypertext history of Instructional Design. Web site. 
Restrepo. D. y Cárdenas, R. Caracterización de la internacionalización del currículo contable...

Bolonia. (1999). Espacio Europeo de Enseñanza Superior. Declaración conjunta de los ministros europeos de enseñanza. Recuperado de: http:/www.unex.es/unex/oficinas/ oce/archivos/ficheros/documentos/ministros/bolonia99.pdf [28/11/2019].

Bloom, B; Hastings, Tomas y Madaus, George F. (1976). Manual de evaluación formativa del currículo. Traducción de María Palavicino V. Colombia: Continental Gráfica.

Bruner, J. (1961). Actual Minds, Possible Worlds. M.A.: Harvard UniversityPress, Cambridge.

Cárdenas, L.R. (2016). Cultura del conocimiento, una búsqueda que da sentido. Primera edición. Medellín: Editorial L. Vieco S.A.S.

Carr y Kemmis (1986). Action Research and Social Movement: A Challenge for Policy Research. College of Education, Arizona State University.

Consejo Nacional de Acreditación -CNA- (2013). Lineamientos para la Acreditación de Programas de Pregrado. Bogotá: Recuperado de: https://www.cna.gov.co/1741/ articles-186359_pregrado_2013.pdf

Declaración de Salamanca. (1994). Marco de Acción para las necesidades educativas especiales, aprobada por la conferencia Mundial sobre necesidades educativas especiales: Acceso y Calidad. Salamanca: Impreso en la UNESCO.

Diccionario de La Real Academia de la Lengua Española (2019). Edición del tricentenario. Recuperado de: https://dle.rae.es/armonizaci\%C3\%B3n

Eliott. (1980). Diseñando el futuro. Textos de la Open University. Nigel Cross.

De Zan, A., Paipa Galeano, L. A., \& Parra Moreno, C. (2011). Las competencias: base para la internacionalización de la educación superior. Revista Educación En Ingeniería, 6(11), 44-54. https://doi.org/10.26507/rei.v6n11.113

Guido, E. y Guzmán Aguilar, A. (2012). Criterios para internacionalizar el currículum universitario. Revista actualidades investigativas en educación, 26. pp. 1-25

Hawawini, G. (05 de 11 de 2011). papers ssrn. Recuperado de https://papers.ssrn.com/sol3/ papers.cfm?abstract_id $=1954697$

Haug, G. (2010). Espacio Europeo de Educación Superior por la puesta en marcha: -del programa Erasmus- del Proceso de Bolonia para la convergencia universitaria; -y de la agenda de la UE por la modernización y competitividad universitaria. Comisión Europea, Parlamento Europeo, OCDE, Banco Mundial, UNESCO. Universidad Politécnica de Valencia España.

Habermas, J. (1973). Theory and Practice. Boston: Beacon Press.

Habermas, J. (1979). Communication and the Evolution of Society. Boston: Beacon Press.

Jackson, W. (1968). La vida en las aulas. Madrid: Morata.

Gacel, R. (09 de 02 de 2008). Universidad Autónoma de México. Recuperado de http:// www.uam.mx/difusion/casadeltiempo/09_iv_jul_2008/casa_del_tiempo_eIV_ num09_02_08.pdf

Gadamer, H, (1975). Truth and method. New York: Seabury.

Gadamer, H. (1979). The problem of historical consciousness. In P. Rabinow \& W. Sullivan (Eds). Interpretative Social Science. London Tavistock.

Galeano, J. (2016). El currículo sitiado: un plan visionario para la innovación del currículo en la región. 1a edición. Medellín: Ojja Publicidad y Mercadeo Ltda.

Gauntier, P. (1963). Practical reasoning: the structure and foundations of prudential and moral arguments and their exemplification in discourse. Londres: Oxford University Press. 
González, A. E. M; Echeverri R. C.; Galindo C. L. A.; Duque Q. S. P.; Vergara I. G. B. (2008). Acerca de un sistema de evaluación por competencias en la formación por ciclos propedéuticos. 1ra edición. Secretaría de educación del Municipio de Medellín. Medellín: Universidad de Antioquia.

Goodson. (2000). El cambio en el curriculum. Barcelona: Editorial octaedro.

Lisboa. (2002). A la luz del Tratado de Lisboa: génesis y evolución del entramado institucional de la Unión Europea. Lisboa: OASIS.

Meyer, R. (1961). The Middle-Irish version of the story of Troy. Of the Michigan Academy of Science, Arts, and Letters.

Ortiz, A. (2015). Internacionalización del currículo. CERI, 38. pp.1-34.

Pérez, A.J. (1983). Paradigmas contemporáneos de investigación didáctica. En J. Gimeno Sacristán y A.J. Pérez Gómez, La enseñanza, su teoría y su práctica. Madrid: Akal.

Ramírez, J. (08 de 08 de 2013). Universidad Libre. Recuperado de: http://www.unilibre.edu. co/bogota/pdfs/2016/3sin/B31.pdf

Reid. W.A. (1985). Curriculum design and moral judgment. Cambrindge journal of education, Vol.3. No 1.pp. 3-7.

Schön, D. (1992). La formación de profesionales reflexivos. Hacia un nuevo diseño de la enseñanza y el aprendizaje en las profesiones. Barcelona: Editorial Paidós.

Schwab, J. (1969). The practical: a language for curriculum. Washington: National Education Association.

Stenhouse, L. (1975). Introduction to curriculum research and development. Heinemann, Londres. Madrid Moratas.

Tangelson, G. (15 de 04 de 2014). Desde el sur: miradas sobre la internacionalización. Recuperado de: Red de Universidades Nacionales del Conurbano Bonaerense: http:// www.unla.edu.ar/images/stories/edunla/desde_sur.pdf\# page $=29$

Taba, H. (1991). Elaboración del currículo, teoría y práctica. Novena edición. Buenos Aires: Troquel.

TYLER Federich W. (1971). Curriculum in International Contexts: Understanding Colonial, Ideological. Canadá: Editorial Springer.

TYLER, Raph (1949). Principios básicos del currículum. Buenos Aires: Troquel.

TYLER, Raph (1981). Specif approaches to curriculum development. En Giroux, H.; Penna, A. y Pinnar, W. (Eds.). Curriculum and Instruction. Berkeley: McCutchan

Yin, R.K. (2009). Case Study Research - Design and Methods, Applied Social Research Methods. (Vol. 5, 2nd ed.), Newbury Park, CA, Sage. 